\title{
Time- and Frequency-Domain Characteristics of Atrial Electrograms During Sinus Rhythm and Atrial Fibrillation
}

\author{
SHENG-HSIUNG CHANG, M.D., MAGNUS ULFARSSON, PH.D., * AMAN CHUGH, M.D., \\ KENTARO YOSHIDA, M.D., KRIT JONGNARANGSIN, M.D., THOMAS CRAWFORD, M.D., \\ ERIC GOOD, D.O., FRANK PELOSI, JR., M.D., FRANK BOGUN, M.D., FRED MORADY, M.D., \\ and HAKAN ORAL, M.D.
}

From the Division of Cardiovascular Medicine, University of Michigan, Ann Arbor, Michigan; and *Department of Electrical and Computer Engineering, University of Iceland, Reykjavik, Iceland

\begin{abstract}
Ablation and Spectral Characteristics of Fibrillation. Background: Complex fractionated atrial electrograms (CFAE) have been considered to be helpful during catheter ablation of atrial fibrillation (AF). The purpose of this study was to analyze the characteristics of CFAEs recorded during sinus rhythm (SR) and $\mathrm{AF}$, and to determine their relationship to perpetuation of $\mathrm{AF}$ and clinical outcome.

Methods and Results: Antral pulmonary vein isolation (APVI) was performed in 34 consecutive patients (age $=59 \pm 10$ years) with paroxysmal AF who presented in SR. Time- and frequency-domain characteristics of electrograms recorded from the same sites in the coronary sinus (CS) were analyzed during SR and $\mathrm{AF}$, before and during isoproterenol infusion. There was a modest correlation in fractionation index (FI: change in the direction of depolarization, $r=0.40, P=0.001$ ) and complexity index (CI: change in the polarity of depolarization, $r=0.41, P=0.001$ ), but not in the dominant frequency (DF) between SR and AF. There was no relationship between the DF and CI or FI during AF. Isoproterenol was associated with an increase in DF during AF $(6.6 \pm 0.9$ vs $5.1 \pm 0.6 \mathrm{~Hz}, \mathrm{P}<0.001)$ but had no effect on $\mathrm{CI}$ or $\mathrm{FI}(\mathrm{P}=$ 0.6). A higher CI $(58.3 \pm 21.0 / \mathrm{s}$ vs $38.0 \pm 21.0 / \mathrm{s}, \mathrm{P}<0.01)$, and FI $(123.5 \pm 44.8 / \mathrm{s}$ vs $75.6 \pm 44.6 / \mathrm{s}, \mathrm{P}<$ 0.01) during AF were associated with a lower likelihood of termination of AF during APVI and a higher probability of recurrent AF after ablation. Ratio of FI during AF to SR was also higher when AF persisted than terminated after APVI $(29.7 \pm 12.4$ vs $19.1 \pm 9.7, \mathrm{P}=0.002)$. However, time- or frequency-domain parameters during SR were not predictive of termination or clinical outcome.

Conclusions: Structural and functional properties of the atrial myocardium during AF contribute to electrogram complexity, which may indicate the presence of extra-PV mechanisms of AF that are not eliminated by APVI. Mapping of complex electrograms in SR is not likely to be sufficient to identify drivers of AF. (J Cardiovasc Electrophysiol, Vol. 22, pp. 851-857, August 2011)
\end{abstract}

atrial fibrillation, complex fractionated atrial electrograms, catheter ablation, spectral analysis, dominant frequency

Complex fractionated atrial electrograms (CFAEs) have been considered helpful to identify target sites in both atria and coronary sinus (CS) during catheter ablation of atrial fibrillation (AF). ${ }^{1-4}$ CFAEs are thought to indicate sites of conduction slowing or block, wave-front collision, anchor points for reentrant circuits, sites of wave-break and fibrillatory conduction at the periphery of high frequency sources, and autonomic innervation. ${ }^{5-8}$ Electrogram fractionation and complexity may also develop due to the structural properties of the underlying tissue that cause summation of activation from overlapping muscle fibers or anisotropy. ${ }^{9}$ It is not clear

This study was supported in part by a grant from St. Jude Medical Inc.

Drs. Oral and Morady report serving on the advisory board of or as a consultant to Medtronic/Ablation Frontiers. Other authors: No disclosures.

Address for correspondence: Hakan Oral, M.D., Cardiovascular Center, SPC 5853, 1500 East Medical Center Drive, Ann Arbor, MI 48109, USA. Fax: 734936 7026; E-mail: oralh@umich.edu

Manuscript received 28 July 2010; Revised manuscript received 26 November 2010; Accepted for publication 23 December 2010.

doi: $10.1111 / \mathrm{j} .1540-8167.2011 .02022 . \mathrm{x}$ whether CFAEs due to structural or functional properties play a critical role in the genesis of AF. The relationship between electrogram complexity during sinus rhythm (SR) and AF also remains unclear.

The purpose of this study was to investigate the characteristics and mechanisms of CFAEs in paroxysmal AF by examining the time- and frequency-domain characteristics of electrograms recorded in the CS during SR and AF before and during infusion of isoproterenol and their relationship to elimination of $\mathrm{AF}$ during ablation.

\section{Methods}

\section{Study Subjects}

The subjects of this study were 34 consecutive patients with paroxysmal AF who presented to the electrophysiology laboratory in SR for de novo radiofrequency catheter ablation of AF. The mean age of the patients was $59 \pm 10$ years. There were 28 men and 6 women. AF was first diagnosed $7 \pm$ 6 years prior to referral. The mean left ventricular ejection fraction was $0.56 \pm 0.07$ and the mean left atrial diameter was $42 \pm 7 \mathrm{~mm}$. Structural heart disease was present in 14 patients $(41 \%)$ : hypertensive heart disease in 11 , coronary 
TABLE 1

Clinical Characteristics

\begin{tabular}{|c|c|c|c|c|c|c|}
\hline & \multicolumn{2}{|c|}{ Termination of AF during RFA } & \multirow[b]{2}{*}{$\mathbf{P}$} & \multicolumn{3}{|c|}{ Freedom from $\mathrm{AF}$} \\
\hline & No $(N=11)$ & Yes $(\mathrm{N}=23)$ & & No $(N=11)$ & Yes $(\mathrm{N}=23)$ & $\overline{\mathbf{P}}$ \\
\hline Age (years) & $55 \pm 14$ & $62 \pm 6$ & 0.1 & $56 \pm 8$ & $61 \pm 10$ & 0.2 \\
\hline Male/female & $9 / 2$ & $19 / 4$ & 1.0 & $10 / 1$ & $18 / 5$ & 0.4 \\
\hline Duration of AF (years) & $7.7 \pm 6.9$ & $6.7 \pm 6.4$ & 0.7 & $6.9 \pm 6.5$ & $7.2 \pm 6.7$ & 0.9 \\
\hline Structural heart disease & $4(36)$ & $10(43)$ & 0.7 & $7(50)$ & $7(35)$ & 0.5 \\
\hline Hypertension & 3 & 8 & & 6 & 5 & \\
\hline Coronary artery disease & 0 & 2 & & 1 & 1 & \\
\hline Nonischemic CM & 1 & 1 & & 1 & 1 & \\
\hline Amiodarone before RFA & 2 & 3 & 0.6 & 2 & 3 & 1.0 \\
\hline Left atrial diameter (mm) & $42 \pm 7$ & $43 \pm 8$ & 0.7 & $40 \pm 4$ & $44 \pm 8$ & 0.1 \\
\hline LV ejection fraction (\%) & $55 \pm 6$ & $57 \pm 9$ & 0.5 & $55 \pm 7$ & $56 \pm 7$ & 0.5 \\
\hline
\end{tabular}

Data are shown as mean \pm SD. Percent values are shown in parentheses. Freedom from AF was assessed after a single ablation procedure. AF $=$ atrial fibrillation; $\mathrm{RFA}=$ radiofrequency catheter ablation; $\mathrm{LV}=$ left ventricle; $\mathrm{CM}=$ cardiomyopathy.

artery disease in 2, and a nonischemic cardiomyopathy in 2 patients (Table 1).

\section{Electrophysiologic Study and Radiofrequency Catheter Ablation}

All patients provided informed written consent for this study. An electrophysiological study was performed in the fasting state under conscious sedation using midazolam and fentanyl. Treatment with antiarrhythmic medications, except for amiodarone in 2 patients, was discontinued 5 half-lives before the procedure. Treatment with amiodarone was discontinued 8 weeks prior to the procedure. Vascular access was obtained through a femoral vein. A decapolar (Biosense Webster, Diamond Bar, CA, USA) catheter with $2.5 \mathrm{~mm}$ interelectrode spacing was positioned in the CS and used for recording electrograms and atrial pacing. The position of the CS catheter was confirmed periodically by fluoroscopy throughout the procedure. Because the CS catheter has a stable position throughout the procedure, electrograms recorded from the distal and middle CS were analyzed in this study. After the transseptal puncture, systemic anticoagulation was achieved with intravenous heparin to maintain an activated clotting time of 300-350 seconds. The pulmonary veins (PVs) were mapped with a decapolar ring catheter (Lasso, Biosense Webster). An open-irrigation, 3.5-mm-tip deflectable catheter (Thermocool, Biosense Webster) was used for mapping and ablation. Bipolar electrograms were displayed at filter settings of $30-500 \mathrm{~Hz}$ during the procedure (EPMed Systems). Electrograms also were recorded at a bandpass of $0.5-200 \mathrm{~Hz}$ for off-line spectral analysis.

An electroanatomical mapping system (Carto, Biosense Webster) was used to construct the 3-dimentional geometry of the left atrium and PVs Antral pulmonary vein isolation (APVI) was performed to isolate all PVs Radiofrequency energy applications were delivered at a maximum power of $35 \mathrm{~W}$ at a flow rate of $30 \mathrm{~mL} / \mathrm{min}$ and a maximum temperature of $48^{\circ} \mathrm{C}$. Near the ostium of the PVs and on the posterior left atrium, radiofrequency energy was delivered at a maximum power of $25 \mathrm{~W}$ at a flow rate of $17 \mathrm{~mL} / \mathrm{min}$.

\section{Study Protocol}

The study protocol was approved by the Institutional Review Board. AF was induced by either isoproterenol infusion up to $20 \mu \mathrm{g} / \mathrm{min}$ (26 patients) or rapid atrial pacing from the
CS (8 patients) prior to ablation. Electrograms from the CS were recorded for 60 seconds: (1) during sinus rhythm at baseline; (2) during isoproterenol infusion at the highest infusion rate both in SR and AF (60 seconds of induction); and (3) during $\mathrm{AF} \geq 10$ minutes after isoproterenol washout or after $\mathrm{AF}$ induction by using rapid atrial pacing from the CS.

Electrograms recorded from the CS were processed and analyzed off-line in the MatLab environment (MathWorks, Natick, MA, USA) using custom software as described previously..$^{10}$ All data and electrograms were reviewed manually and appropriateness of detection was validated. Electrogram analysis was performed during SR and $\mathrm{AF}$ as follows:

(i) Electrogram analysis during SR: Bipolar electrograms with the largest amplitude in each 10-second recording sample were used for analysis. The dominant frequency (DF) was defined as the frequency of the highest peak of the periodogram using Fast Fourier Transformation. The spectral pattern was categorized as: Type 1- a single frequency component constituting $>90 \%$ of the total power; or Type 2- multiple frequency components, each with $\geq 20 \%$ of the maximum power (Fig. 1). The characteristics of atrial electrograms were also analyzed in the time-domain: (1) duration of the atrial electrogram, measured from the earliest to the latest atrial electrogram deflection; (2) amplitude, measured as the voltage difference between the maximum positive and minimum negative deflection of the electrogram; (3) fractionation index (FI), the number of changes in the direction of depolarization; and (4) complexity index (CI), the number of changes in the polarity of the depolarization (Fig. 1).

(ii) Electrogram Analysis during AF: QRS complexes were subtracted first and spectral and time domain analyses were performed as described previously. ${ }^{10,11}$ In addition to the DF, the mean amplitude was determined as the mean of the maximum 60 electrogram amplitudes in a sampling window, and parameters of fractionation were determined as the frequency of change in the direction of depolarization (FI) and polarity of depolarization (CI) per second (Fig. 1).

The following parameters were systematically analyzed: (1) time- and frequency-domain characteristics during SR and AF; (2) effect of isoproterenol on time- and frequencydomain characteristics during SR and AF; (3) relationship between frequency- and time-domain characteristics; and (4) relationship between time- and frequency-domain characteristics and termination of AF during APVI and clinical outcome after catheter ablation. 


\section{Sinus Rhythm}
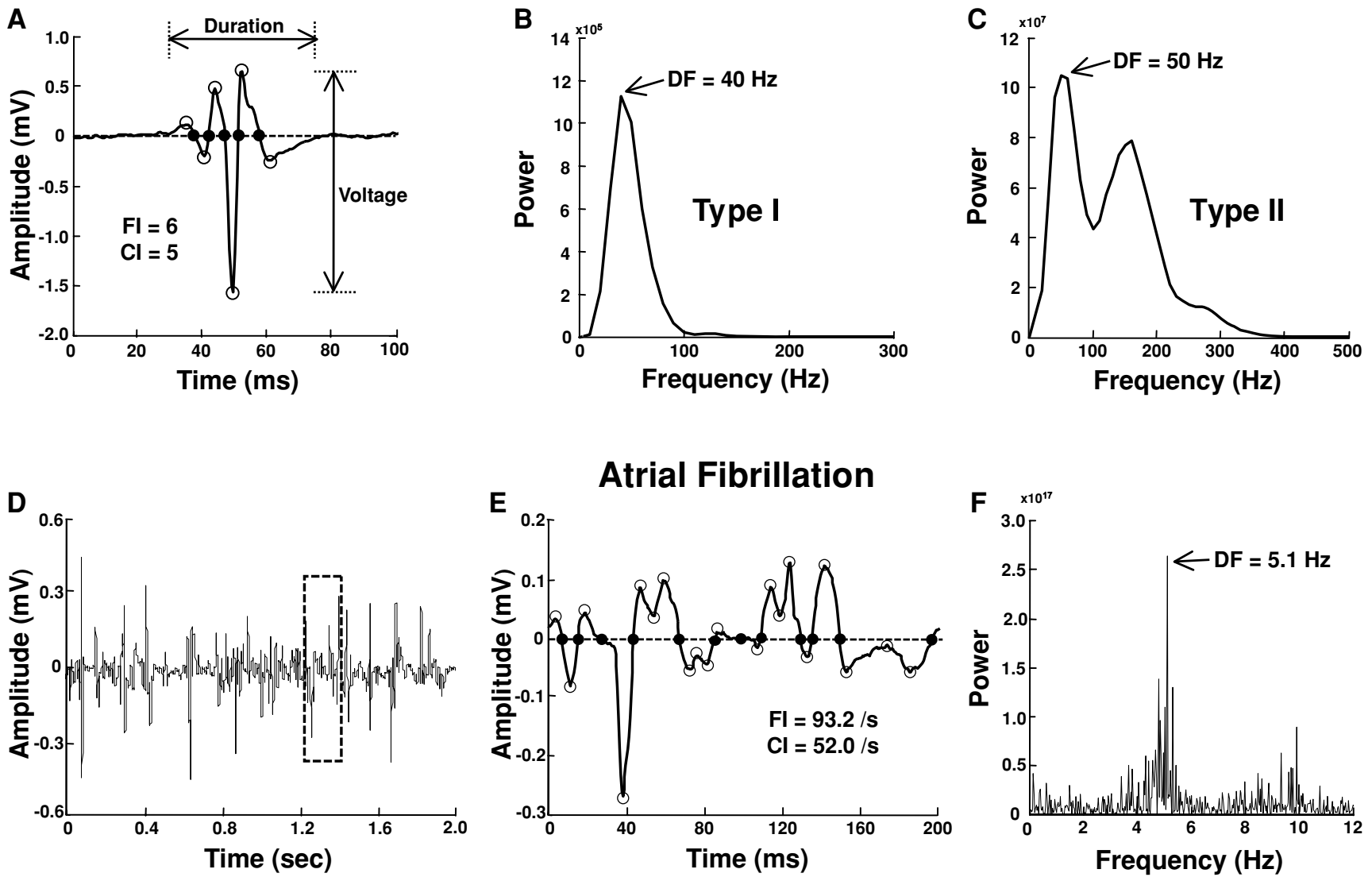

Figure 1. Analysis of atrial electrograms recorded in coronary sinus (CS) during sinus rhythm (SR, Panels A-C) and atrial fibrillation (AF, Panels D-F) in time- and frequency-domains. A single electrogram during SR is shown in Panel (A) and a 2-second recording of electrograms during AF is shown in Panel (D). Boxed area in Panel (D) is enlarged as Panel (E) for better resolution. Fractionation index (FI) was defined as the change in the direction of depolarization (open circles, Panels A and E), whereas complexity index (CI) was defined as the change in the polarity (full circles, Panels A and E). During $S R$, the spectral pattern was categorized as type 1 (Panel B) and type 2 (Panel C) periodograms. Panel $(F)$ shows the periodogram of frequency-domain analysis during $A F . D F=$ dominant frequency.

\section{Post Ablation Care and Follow-Up}

All patients were treated with intravenous heparin after the procedure, and were discharged home the next day on warfarin and low molecular weight heparin until the international normalized ratio was $>2.0$. Patients were first seen in an outpatient clinic 3 months after the ablation and every 6 months thereafter. Patients were contacted periodically by a nurse and were asked to call a clinical coordinator whenever they experienced symptoms suggestive of an arrhythmia. A 30-day auto-triggered event monitor was provided to all patients 6 months after ablation.

A successful clinical outcome was defined as freedom from recurrent atrial arrhythmias ( $\leq 30$ seconds) in the absence of antiarrhythmic drug therapy after a single ablation procedure. The mean duration of follow-up was $13 \pm 5$ months.

\section{Statistical Analysis}

Continuous variables are expressed as mean \pm 1 standard deviation and were compared by Student's $t$-test. Categorical variables were compared by Chi-square analysis or with Fisher's exact test where appropriate. Pearson's correlation coefficients were used to determine the relationship between various parameters. A $\mathrm{P}<0.05$ indicated statistical significance.

\section{Results}

\section{Time- and Frequency-Domain Characteristics During SR}

During SR, the mean number of changes (per electrogram) in the direction (FI) and polarity (CI) of the electrograms were $4.1 \pm 1.3$ and $2.9 \pm 1.1$, respectively. There was a direct correlation between FI and $\mathrm{CI}(\mathrm{r}=0.88, \mathrm{P}<0.001$, Fig. 3). The mean DF during SR was $54.9 \pm 19.4 \mathrm{~Hz}$. There was a statistically significant direct linear correlation between the $\mathrm{DF}$ and $\mathrm{CI}(\mathrm{r}=0.31, \mathrm{P}=0.01)$, but not between the DF and $\mathrm{FI}(\mathrm{r}=0.18, \mathrm{P}=0.14)$.

The mean amplitude and duration of the electrograms were $2.9 \pm 2.0 \mathrm{mV}$ and $39.7 \pm 9.0 \mathrm{~ms}$, respectively. There was a negative correlation between the amplitude and FI $(\mathrm{r}=$ $-0.30, \mathrm{P}=0.01$, Fig. 3 ) and between the amplitude and duration $(\mathrm{r}=-0.35, \mathrm{P}=0.004)$, but not between the amplitude and $\mathrm{DF}(\mathrm{r}=0.22, \mathrm{P}=0.08)$ or between the amplitude and CI $(r=-0.21, P=0.10)$. 
A

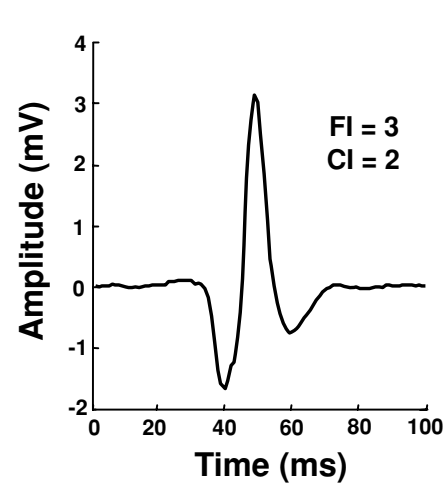

Sinus Rhythm

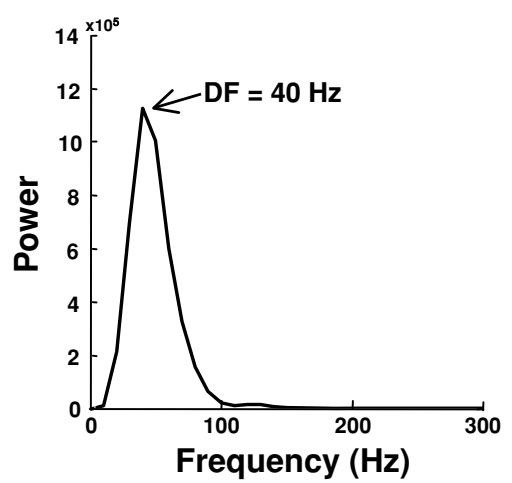

Sinus Rhythm

B
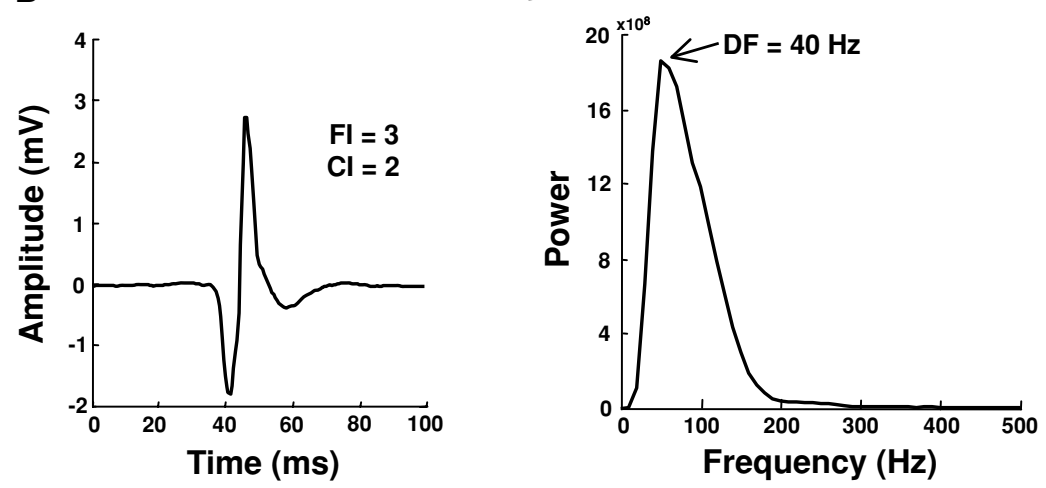

Atrial Fibrillation

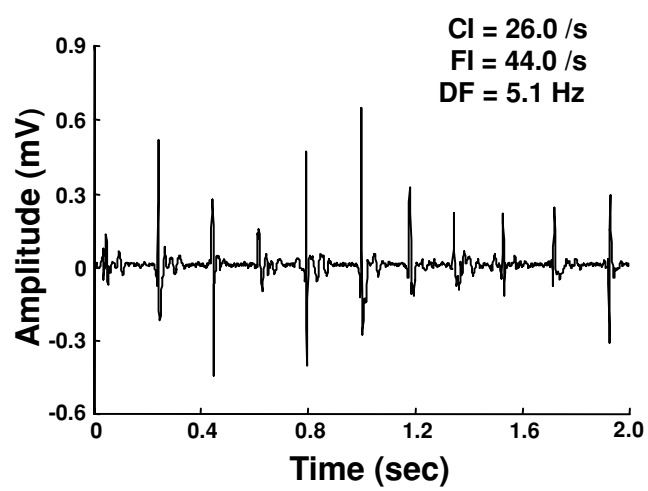

Atrial Fibrillation

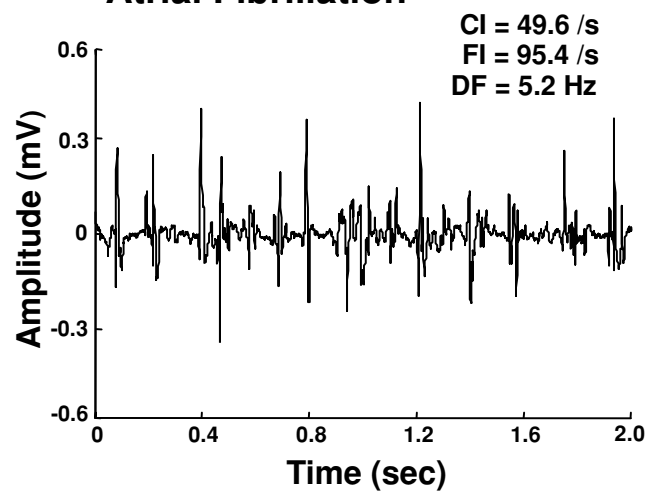

Figure 2. Time- and frequency-domain characteristics of CS electrograms in 2 patients who did not (Panel A) and did (Panel B) have recurrent AF after APVI. In SR (left panel) electrogram characteristics were similar between the 2 patients. However, during AF, FI, and CI were higher in the patient who had recurrent AF after APVI. Abbreviations as in Figure 1.

\section{Time- and Frequency-Domain Characteristics During AF}

During AF, FI and CI were $90.1 \pm 49.5 / \mathrm{s}$ and $44.1 \pm$ $22.9 / \mathrm{s}$, respectively, at baseline. There was a direct correlation between FI and CI during $\mathrm{AF}(\mathrm{r}=0.96, \mathrm{P}<0.001$, Fig. 3). The mean DF was $5.2 \pm 0.7 \mathrm{~Hz}$. There was no correlation between the DF and FI $(r=0.17, \mathrm{P}=0.2)$ or between the DF and $\mathrm{CI}(\mathrm{r}=0.22, \mathrm{P}=0.07)$.

The mean amplitude was $0.58 \pm 0.33 \mathrm{mV}$. There was a negative correlation between the amplitude and CI $(\mathrm{r}=$ $-0.55, \mathrm{P}<0.001)$, and between the amplitude and FI $(\mathrm{r}=$ $-0.55, \mathrm{P}<0.001$, Fig. 3). There was no significant relationship between the amplitude and DF $(\mathrm{r}=-0.04, \mathrm{P}=$ $0.78)$.

\section{Relationship between Time- and Frequency-Domain Parameters During SR and AF}

There was a direct relationship between the amplitude $(\mathrm{r}=$ $0.44, \mathrm{P}<0.001), \mathrm{FI}(\mathrm{r}=0.40, \mathrm{P}=0.001)$ and $\mathrm{CI}(\mathrm{r}=0.41$, $\mathrm{P}=0.001)$ during $\mathrm{SR}$ and AF. There was no relationship between DF during $\mathrm{SR}$ and $\mathrm{AF}(\mathrm{r}=0.01, \mathrm{P}=0.96)$.

During SR, sites that displayed a type 2 periodogram had a longer duration $(43.7 \pm 10.0$ vs $36.4 \pm 6.4, \mathrm{P}=0.001)$, higher FI ( $4.4 \pm 1.7$ vs $3.8 \pm 0.7, \mathrm{P}=0.045)$ and $\mathrm{CI}(3.2 \pm$ 1.5 vs $2.6 \pm 0.7, \mathrm{P}=0.045)$, but a lower amplitude ( $2.07 \pm$ 1.14 vs $3.60 \pm 2.30, \mathrm{P}=0.002)$ in the time-domain than sites that displayed a type 1 periodogram. However, during
AF, there was no difference in FI $(85.7 \pm 45.7 / \mathrm{s}$ vs $95.4 \pm$ $54.2 / \mathrm{s}, \mathrm{P}=0.4), \mathrm{CI}(41.8 \pm 19.7 / \mathrm{s}$ vs $46.9 \pm 26.2 / \mathrm{s}, \mathrm{P}=$ $0.4)$ or amplitude $(0.58 \pm 0.32$ vs $0.58 \pm 0.35, \mathrm{P}=0.9)$ between sites with a type 1 and 2 periodogram. The DF was also similar between sites with a type 1 or 2 periodogram during both $\mathrm{SR}$ and $\mathrm{AF}(\mathrm{P}=0.3$ and $\mathrm{P}=0.6$, respectively).

\section{Effect of Isoproterenol}

Isoproterenol infusion resulted in a decrease in the SR cycle length from $990 \pm 161 \mathrm{~ms}$ to $583 \pm 143 \mathrm{~ms}(\mathrm{P}<$ $0.001)$ and an increase in amplitude from $2.59 \pm 1.70 \mathrm{mV}$ to $3.88 \pm 2.89 \mathrm{mV}(\mathrm{P}<0.001)$. There was no change in the $\mathrm{DF}$ during SR $(55.7 \pm 21.2 \mathrm{~Hz}$ vs $56.7 \pm 24.2 \mathrm{~Hz}, \mathrm{P}=0.6)$ or duration of electrograms $(39.4 \pm 8.8 \mathrm{~ms}$ vs $38.2 \pm 8.7 \mathrm{~ms}$, $\mathrm{P}=0.2$ ). Isoproterenol did not have an effect on FI or CI during SR ( $\mathrm{P}=0.1$ and 0.4 , respectively).

During AF, isoproterenol resulted in an increase in the amplitude $(0.67 \pm 0.45 \mathrm{mV}$ vs $0.53 \pm 0.33 \mathrm{mV}, \mathrm{P}=0.01)$ and $\mathrm{DF}(6.6 \pm 0.9 \mathrm{~Hz}$ vs $5.1 \pm 0.6 \mathrm{~Hz}, \mathrm{P}<0.001)$, but did not have an effect on FI and $\mathrm{CI}(\mathrm{P}=0.8$ and 0.6 , respectively). There was no difference in DF $(6.6 \pm 0.9 \mathrm{~Hz}$ vs $6.6 \pm$ $0.9 \mathrm{~Hz}, \mathrm{P}=0.9)$, FI $(87.4 \pm 38.0 / \mathrm{s}$ vs $109.6 \pm 52.6 / \mathrm{s}$, $\mathrm{P}=0.1), \mathrm{CI}(45.3 \pm 17.0 / \mathrm{s}$ vs $53.4 \pm 25.9 / \mathrm{s}, \mathrm{P}=0.2)$, or amplitude $(0.70 \pm 0.43$ vs $0.63 \pm 0.49, \mathrm{P}=0.6)$ between sites with a type 1 and 2 periodogram during isoproterenol infusion. 
Figure 3. Relationship between the fractionation and complexity indices, and between amplitude and the fractionation index during SR (Panel A) and AF (Panel B). Abbreviations as in Figure 1.
A

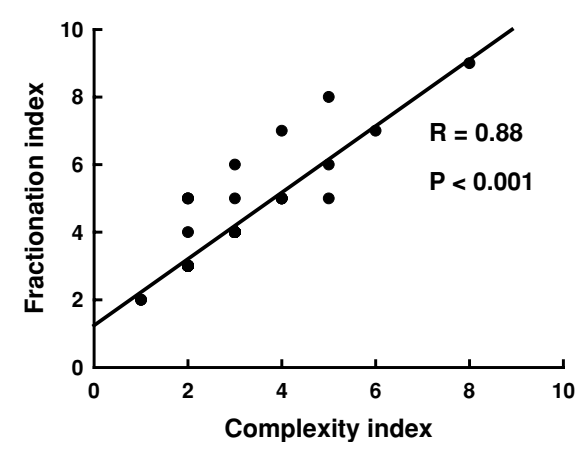

B

Sinus Rhythm

Atrial Fibrillation

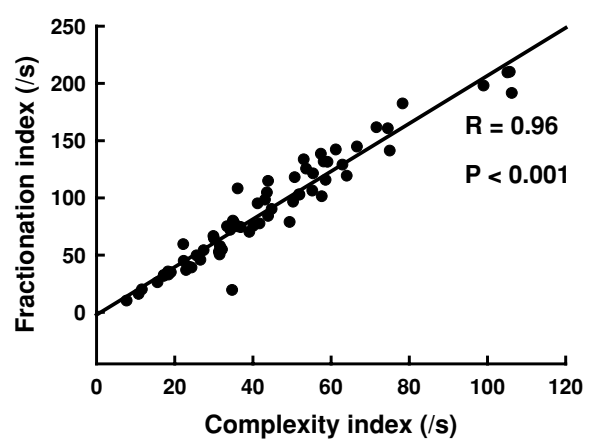

Sinus Rhythm

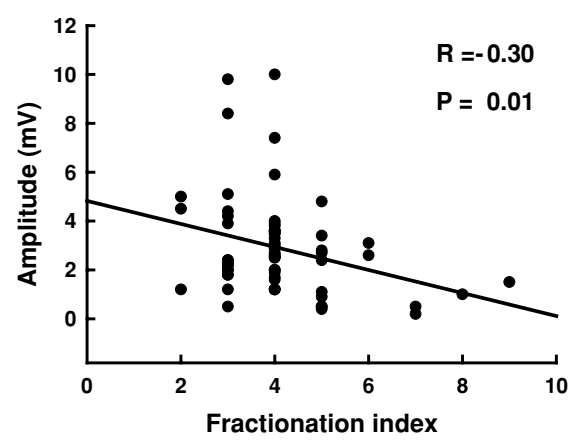

Atrial Fibrillation

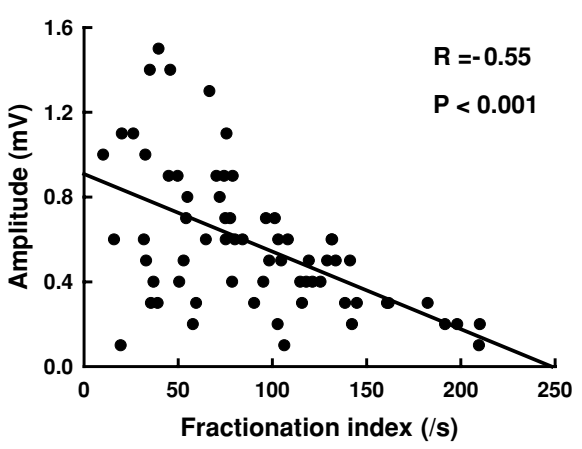

\section{Time- and Frequency-Domain Parameters and Termination of AF during Ablation}

$\mathrm{AF}$ terminated in 23 of 34 patients (68\%) during ablation: to sinus rhythm in 21 and to an atrial tachycardia/flutter in 2 . There was no difference in clinical characteristics among patients in whom AF did and did not terminate during ablation (Table 1).

During SR, prior to ablation, the amplitude, duration, DF, spectral pattern (type 1 or 2), FI and CI were similar among patients who did and did not have termination of AF during ablation (Table 2).

During AF, before ablation, the DF was also similar between patients in whom AF did and did not terminate during APVI. However, the mean FI and CI were lower and the amplitude was higher in patients who had termination of $\mathrm{AF}$ than who did not (Table 2).

To determine the effect of AF on fractionation and complexity of electrograms, FI and CI during SR and AF were compared. Patients who did not have termination of AF during ablation had a higher ratio of FI during AF to FI during $\mathrm{SR}\left(\mathrm{FI}_{\mathrm{AF} / \mathrm{SR}}\right)$ than patients in whom AF terminated (29.7 \pm 12.4 vs $19.1 \pm 9.7, \mathrm{P}=0.002$ ). There also was a higher ratio of $\mathrm{CI}$ during $\mathrm{AF}$ to $\mathrm{CI}$ during $\mathrm{SR}\left(\mathrm{CI} \mathrm{AF}_{\mathrm{AR}}\right)$ in patients who did not have termination of AF during ablation than who did $(19.9 \pm 9.1$ vs $14.5 \pm 6.9, \mathrm{P}=0.03)$.

\section{Fractionation and Complexity During SR and AF and Clinical Outcome}

During a mean follow-up of $13 \pm 5$ months after a single ablation procedure, 20 patients (59\%) were in sinus rhythm in the absence of antiarrhythmic drug therapy. Among the 14 patients with recurrent atrial arrhythmias, 11 had $\mathrm{AF}$ and 3 had atrial tachycardia or flutter. There was no difference in clinical characteristics among patients who did and did not remain free from recurrent $\mathrm{AF}$ after the ablation (Table 1).

The FI and CI were higher during AF, but not during SR, in patients who had recurrent AF than who did not (Table 2 and Fig. 2). Patients who had recurrent AF also had a higher $\mathrm{FI}_{\mathrm{AF} / \mathrm{SR}}$ than who did not $(26.4 \pm 11.5$ vs $20.2 \pm 11.2, \mathrm{P}=$ $0.04)$.

\section{Discussion}

\section{Main Findings}

The main findings of this study are: (1) there is a direct correlation between change in the direction (fractionation index) and change in the polarity (complexity index) of electrograms during SR and AF; (2) there is an inverse correlation between the amplitude and CI and FI of electrograms in SR and AF; (3) there is no relationship between DF and $\mathrm{CI}$ or FI of electrograms in AF; (4) there is a modest direct relationship in amplitude, FI and CI (but not DF) between electrograms recorded in SR and in AF; (5) isoproterenol is associated with an increase in amplitude both in SR and AF, and an increase in DF only during AF; (6) FI and CI are lower among patients who are more likely to remain in SR after APVI; and (7) type I or II electrograms are not predictive of complexity of electrograms or DF or clinical outcome after APVI. 
TABLE 2

Characteristic CS Electrograms

\begin{tabular}{|c|c|c|c|c|c|}
\hline & & \multicolumn{2}{|c|}{ Termination of AF During RFA } & \multicolumn{2}{|c|}{ Freedom from $A F$} \\
\hline & & No $(n=20)$ & Yes $(n=46)$ & No $(n=22)$ & Yes $(n=44)$ \\
\hline Duration (ms) & SR & $39.7 \pm 8.6$ & $39.8 \pm 9.2$ & $40.0 \pm 8.6$ & $39.6 \pm 9.2$ \\
\hline Type 2 spectral pattern & SR & $7(35)$ & $23(50)$ & $9(41)$ & $21(48)$ \\
\hline \multirow[t]{2}{*}{$\mathrm{DF}(\mathrm{Hz})$} & SR & $57.2 \pm 17.7$ & $53.9 \pm 20.2$ & $52.0 \pm 14.8$ & $56.3 \pm 21.3$ \\
\hline & $\mathrm{AF}$ & $5.2 \pm 0.8$ & $5.2 \pm 0.7$ & $5.1 \pm 0.8$ & $5.2 \pm 0.7$ \\
\hline \multirow[t]{2}{*}{ Amplitude (mV) } & SR & $2.83 \pm 1.69$ & $3.93 \pm 2.14$ & $2.52 \pm 1.27$ & $3.09 \pm 2.27$ \\
\hline & $\mathrm{AF}$ & $0.42 \pm 0.17$ & $0.65 \pm 0.36^{\dagger}$ & $0.54 \pm 0.30$ & $0.60 \pm 0.35$ \\
\hline \multirow[t]{2}{*}{ Fractionation index } & SR & $4.4 \pm 1.1$ & $4.0 \pm 1.3$ & $4.2 \pm 1.0$ & $4.0 \pm 1.4$ \\
\hline & $\mathrm{AF}$ & $123.5 \pm 44.8$ & $75.6 \pm 44.6^{\dagger}$ & $108.9 \pm 48.1$ & $80.7 \pm 04.1^{*}$ \\
\hline \multirow[t]{2}{*}{ Complexity index } & SR & $3.2 \pm 0.9$ & $2.7 \pm 1.2$ & $3.0 \pm 0.8$ & $2.8 \pm 1.3$ \\
\hline & $\mathrm{AF}$ & $58.3 \pm 21.0$ & $38.0 \pm 21.0^{\dagger}$ & $52.1 \pm 25.0$ & $40.1 \pm 20.9^{*}$ \\
\hline
\end{tabular}

Data are shown as mean \pm SD. Percent values are shown in parenthesis. Abbreviations as in Table 1.

$* \mathrm{P}<0.05$.

${ }^{\dagger} \mathrm{P}<0.01$.

Taken together these findings suggest that structural and functional properties contribute to electrogram fractionation and complexity and that fractionation and complexity may indicate the presence of extra-PV mechanisms as perpetuators of AF that are not eliminated by APVI.

\section{Electrogram Complexity During SR and AF}

A finding of this study is that change in the direction (fractionation) and polarity (complexity) of electrograms are highly correlated both in SR and AF. Complex electrograms appear to have smaller amplitudes than other electrograms, possibly due to preexisting fibrosis and multiplicity of directions of wave-front propagation in multiple tissue layers. Change in the direction and polarity of electrograms are most likely due to anisotropy and complex histological organization of the underlying myocardium. ${ }^{12}$ A modest linear relationship between the complexity of electrograms recorded in SR and during AF may suggest that fixed structural and/or electrophysiologic properties of the myocardium contribute to the complexity of the electrograms.

\section{Complexity and DF}

There was no relationship between the DF and complexity of electrograms during AF suggesting that the 2 parameters often used to map drivers of AF indicate different mechanisms of AF. DF is influenced both by the frequency (cycle length) and also amplitude (power) of electrograms analyzed. At a given site, an electrogram component with a large amplitude but a long cycle length is more likely to be identified as the spectral component indicating DF than a small amplitude, complex fractionated electrogram with a short cycle length that may indeed represent a critical driver. Therefore, DF mapping in the absence of simultaneous high-density recordings may not be sufficient to accurately identify critical drivers of AF.

\section{Effect of Isoproterenol on Electrogram Characteristics}

Isoproterenol increased the amplitude of electrograms both in SR and during AF. Isoproterenol is likely to increase the electrochemical gradients across the cell membrane, which facilitates larger action potentials. Isoproterenol was also associated with an increase in DF only during AF; however, it had no effect on complexity and fractionation of electrograms. Isoproterenol may increase DF by a number of mechanisms: (1) an increase in automaticity and frequency of discharges from an automatic focus; ${ }^{13}$ (2) a decrease in effective refractory period and action potential duration; ${ }^{14}$ or (3) an increase in conduction velocity leading to a shorter cycle length. ${ }^{15}$

Because there was no increase in the complexity and fractionation indices despite an increase in DF during isoproterenol infusion, it may be that complexity and fractionation of electrograms are primarily due to preexisting structural properties rather than due to functional response to a higher activation rate that otherwise would be expected to promote wave-break, fractionation and fibrillatory conduction. ${ }^{6,16}$ However, it should be noted that isoproterenol also increases the conduction velocity and may have led to an increase in the threshold activation frequency at which wavebreak occurs. Therefore, the effects of isoproterenol on DF and conduction velocity may have negated a net change in complexity indices. In addition, an isoproterenol-induced increase in activation rate in this study may not have reached the critical threshold above which further fibrillatory conduction develops.

\section{Electrogram Complexity, Termination of AF and Clinical Outcome}

Consistent with the findings of a prior study, CI and FI were lower when AF terminated than when AF persisted during APVI. ${ }^{10}$ Furthermore, electrogram amplitude was larger in patients who had termination of AF than in whom AF persisted. However, baseline DF was not predictive of termination of AF during AF. It is likely that APVI eliminates high frequency drivers (high DF) that are likely to reside within the PVs and their antral regions. However, complex and fractionated electrograms may indicate mechanisms of AF likely to be reentrant, localized outside the PVs, and not eliminated by APVI. Therefore, a higher prevalence of complex electrograms in patients in whom AF persists after APVI may indicate extra-PV mechanisms that contribute to perpetuation of AF. This may also explain why complexity and fractionation (but not DF) of electrograms recorded in 
the coronary sinus was predictive of recurrent AF after APVI in patients with paroxysmal AF.

In an effort to assess the role of functional effects of $\mathrm{AF}$ on electrogram complexity and fractionation, the ratio of CI and FI in AF to SR was compared among patients who did and did not have termination of AF during APVI. The $\mathrm{FI}_{\mathrm{AF} / \mathrm{SR}}$ and $\mathrm{CI}_{\mathrm{AF} / \mathrm{SR}}$ were higher among patients whose AF persisted than terminated during ablation. This observation may suggest that functional and/or structural changes in the myocardium that develop or become manifest during AF play a critical role in the occurrence of complex electrograms.

\section{Prior studies}

In a prior study, $\mathrm{AF}$ was eliminated by targeting fibrillar myocardium that displayed similar characteristics to type II electrograms in this study. ${ }^{4}$ Unlike in the prior study, there was no relationship between the efficacy of ablation and type of electrograms in this study. However, in this study electrogram analysis was performed only within the CS and specific electrogram types were not targeted for ablation.

\section{Study Limitations}

A limitation of this study is the relatively small sample size. A second limitation is that only CS electrograms were analyzed. Conclusions on the global spatiotemporal organization of AF cannot be made based on the findings of this study. However, electrograms recorded from a fixed and stable multipolar electrode catheter enabled a robust comparison of electrograms recorded from the very same site during SR and AF. Finally, only patients with paroxysmal AF were included in this study; whether these findings can be extrapolated to patients with persistent AF remains to be determined in future studies.

\section{Clinical Implications}

The findings of this study suggest that mapping of complex electrograms during SR is unlikely to be adequate to identify drivers of AF. Rather, it seems necessary to look for complex electrograms during AF. These observations may suggest that functional characteristics of the atrial tissue, in addition to structural properties, play a key role in development of complex electrograms, some of which may become manifest only during AF.

It also appears that mapping both in time- and frequency domains is necessary to identify drivers of AF. Although high frequency focal sources may be better identified using spectral analysis, complex electrograms as identified in time-domain may be more helpful to map reentrant drivers of AF. Nevertheless, time- and frequency-domain characteristics of complex electrograms that indicate specific underlying drivers of AF need to better defined in future prospective studies utilizing high-density multisite mapping and objective parameters to assess electrogram characteristics.

\section{References}

1. Nademanee K, McKenzie J, Kosar E, Schwab M, Sunsaneewitayakul B, Vasavakul T, Khunnawat C, Ngarmukos T: A new approach for catheter ablation of atrial fibrillation: Mapping of the electrophysiologic substrate. J Am Coll Cardiol 2004;43:2044-2053.

2. Oral H, Chugh A, Good E, Wimmer A, Dey S, Gadeela N, Sankaran S, Crawford T, Sarrazin JF, Kuhne M, Chalfoun N, Wells D, Frederick M, Fortino J, Benloucif-Moore S, Jongnarangsin K, Pelosi F Jr, Bogun F, Morady F: Radiofrequency catheter ablation of chronic atrial fibrillation guided by complex electrograms. Circulation 2007;115:2606-2612.

3. Haissaguerre M, Sanders P, Hocini M, Takahashi Y, Rotter M, Sacher F, Rostock T, Hsu LF, Bordachar P, Reuter S, Roudaut R, Clementy J, Jais $P$ : Catheter ablation of long-lasting persistent atrial fibrillation: Critical structures for termination. J Cardiovasc Electrophysiol 2005;16:11251137.

4. Pachon MJ, Pachon ME, Pachon MJ, Lobo TJ, Pachon MZ, Vargas RN, Pachon DQ, Lopez MF, Jatene AD: A new treatment for atrial fibrillation based on spectral analysis to guide the catheter rf-ablation. Europace 2004;6:590-601.

5. Konings KT, Smeets JL, Penn OC, Wellens HJ, Allessie MA: Configuration of unipolar atrial electrograms during electrically induced atrial fibrillation in humans. Circulation 1997;95:1231-1241.

6. Kalifa J, Tanaka K, Zaitsev AV, Warren M, Vaidyanathan R, Auerbach D, Pandit S, Vikstrom KL, Ploutz-Snyder R, Talkachou A, Atienza F, Guiraudon G, Jalife J, Berenfeld O: Mechanisms of wave fractionation at boundaries of high-frequency excitation in the posterior left atrium of the isolated sheep heart during atrial fibrillation. Circulation 2006;113:626-633.

7. Lellouche N, Buch E, Celigoj A, Siegerman C, Cesario D, De Diego C, Mahajan A, Boyle NG, Wiener I, Garfinkel A, Shivkumar K: Functional characterization of atrial electrograms in sinus rhythm delineates sites of parasympathetic innervation in patients with paroxysmal atrial fibrillation. J Am Coll Cardiol 2007;50:1324-1331.

8. Lin J, Scherlag BJ, Zhou J, Lu Z, Patterson E, Jackman WM, Lazzara R, Po SS: Autonomic mechanism to explain complex fractionated atrial electrograms (cfae). J Cardiovasc Electrophysiol 2007;18:1197-1205.

9. Ho SY, Anderson RH, Sanchez-Quintana D: Atrial structure and fibres: Morphologic bases of atrial conduction. Cardiovasc Res 2002;54:325336.

10. Yoshida K, Ulfarsson M, Tada H, Chugh A, Good E, Kuhne M, Crawford T, Sarrazin JF, Chalfoun N, Wells D, Jongnarangsin K, Pelosi F Jr, Bogun F, Morady F, Oral H: Complex electrograms within the coronary sinus: Time- and frequency-domain characteristics, effects of antral pulmonary vein isolation, and relationship to clinical outcome in patients with paroxysmal and persistent atrial fibrillation. J Cardiovasc Electrophysiol 2008;19:1017-1023.

11. Lemola K, Ting M, Gupta P, Anker JN, Chugh A, Good E, Reich S, Tschopp D, Igic P, Elmouchi D, Jongnarangsin K, Bogun F, Pelosi F Jr, Morady F, Oral H: Effects of two different catheter ablation techniques on spectral characteristics of atrial fibrillation. J Am Coll Cardiol 2006;48:340-348.

12. Saito T, Waki K, Becker AE: Left atrial myocardial extension onto pulmonary veins in humans: Anatomic observations relevant for atrial arrhythmias. J Cardiovasc Electrophysiol 2000;11:888-894.

13. Yamazaki M, Vaquero LM, Hou L, Campbell K, Zlochiver S, Klos M, Mironov S, Berenfeld O, Honjo H, Kodama I, Jalife J, Kalifa J: Mechanisms of stretch-induced atrial fibrillation in the presence and the absence of adrenocholinergic stimulation: Interplay between rotors and focal discharges. Heart Rhythm 2009;6:1009-1017.

14. Nof E, Burashnikov A, Antzelevitch C: Cellular basis for atrial fibrillation in an experimental model of short qt1: Implications for a pharmacological approach to therapy. Heart Rhythm 2010;7:251-257.

15. Morady F, Nelson SD, Kou WH, Pratley R, Schmaltz S, De Buitleir M, Halter JB: Electrophysiologic effects of epinephrine in humans. J Am Coll Cardiol 1988;11:1235-1244.

16. Rostock T, Rotter M, Sanders P, Takahashi Y, Jais P, Hocini M, Hsu LF, Sacher F, Clementy J, Haissaguerre M: High-density activation mapping of fractionated electrograms in the atria of patients with paroxysmal atrial fibrillation. Heart Rhythm 2006;3:27-34. 